УДК 550.34

ТЕОРИЯ ПОДОБИЯ ПРОСТРАНСТВЕННОЙ И СТРУКТУРЫ СЕЙСМИЧНОСТИ

${ }^{1}$ Герман В.И.

${ }^{1}$ ГПКК «Красноярский научно-исследовательский институт геологии и минерального сырья» (ГПКК «КНИИГиМС»), 660049, Красноярск, пр. Мира, 55, e-mail: german3v@yandex.ru

Рассмотрена теория масштабирования функций распределения

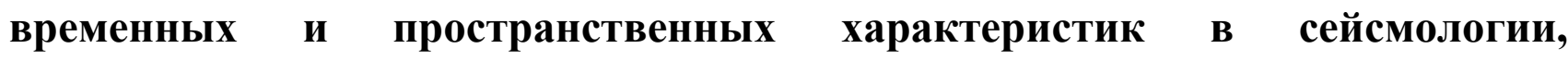
соответствующая предположению о подобии структуры сейсмичности в разных энергетически-пространственно-временных интервалах. Приведен пример ее проверки на данных Токтогульского района (Киргизия).

Ключевые слова: теория подобия, структура сейсмичности

\title{
SCALING THEORY OF SPATIAL AND TEMPORAL SEISMICITY STRUCTURE
}

\begin{abstract}
${ }^{1}$ Victor I. German
${ }^{1}$ Krasnoyarsk Research Institute of Geology and Minerals, 660049, Russia, Krasnoyarsk, Miraav., 55, e-mail: german3v@yandex.ru
\end{abstract}

The scaling theory of the distribution functions of temporal and spatial characteristics in seismology is considered. It corresponds to assumption of the similarity of seismicity structure in the various energy-space-time intervals. An example of its checks on the data of the Toktogul district (Kyrgyzstan) is shown.

Keywords: scaling theory, seismicity structure 


\section{Введение}

Структура сейсмичности, т.е. регистрируемой последовательности сейсмических событий, является основной информацией для понимания физических процессов, идущих в земной коре. Ее изучение является важной научной задачей и необходимо для прогноза сильных сейсмических событий.

Для анализа структуры сейсмичности необходимо зафиксировать энергетическо-пространственно-временной интервал (ЭПВИ), который будет определять набор рассматриваемых сейсмических событий. ЭПВИ задается начальными значениями энергии $E$, координат $X$ и $Y$, времени $T$, а также величин их приращения: $E I, \square E I ; X I, \square X I, Y I, \square Y I, Z I, \square Z I ; T I, \square T I$. В дальнейшем ЭПВИ с такими параметрами будем обозначать $I$.

Вместо параметров EI и $\square$ EI, задающих значения энергии, можно использовать значения магнитуды MI и $\square$ MI.

В качестве основных характеристик сейсмичности в данной работе рассматриваются времена ожидания между хронологически последовательными сейсмическими событиями в ЭПВИ $\Delta \mathrm{T}$ (Рис. 1), а также новая характеристика $\Delta \mathrm{Dmin}$ - минимальное расстояние от текущего события до самого близкого (в пространстве) соседнего события в ЭПВИ (Рис. 2) [3, 10].

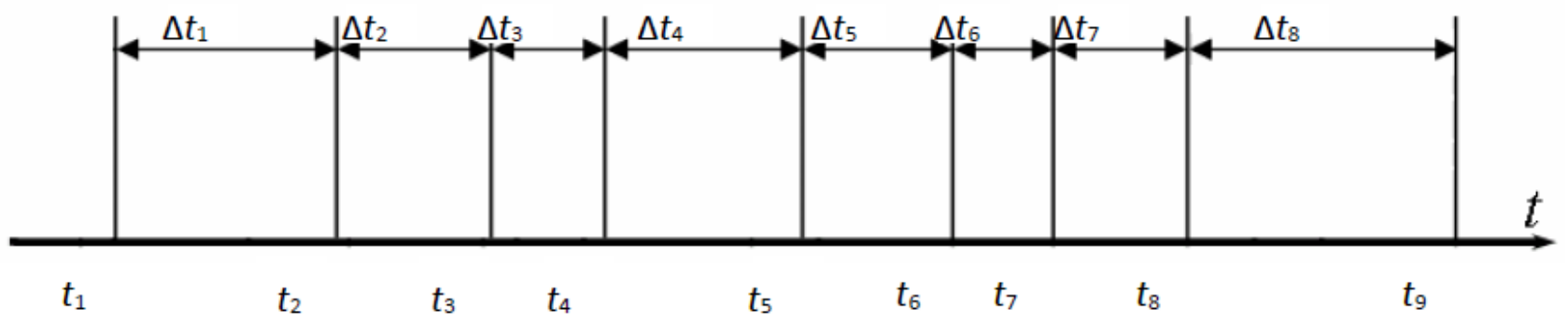

Рис. 1. Времена ожидания сейсмических событий (времена возникновения $\mathrm{t}$ событий отмечены вертикальными линиями) 


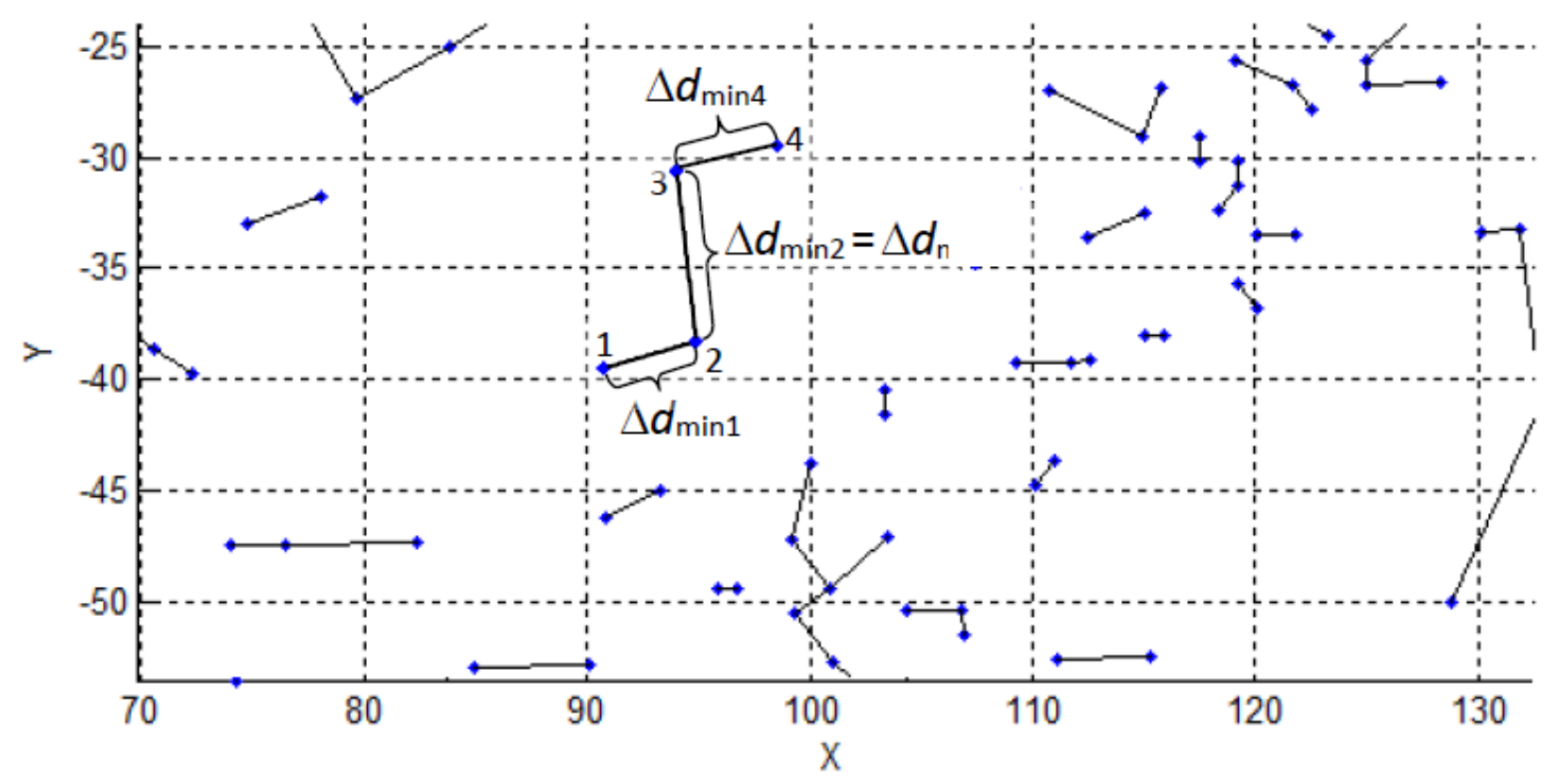

Рис. 2. Минимальные расстояния между сейсмическими событиями $\min \Delta d$ (события обозначены точками, ближайшие события соединены отрезками, их длина соответствует значению $\Delta d_{\min }$ )

Распределения характеристик $\square$ T и $\square$ Dmin позволяют оценивать временной интервал до следующего сейсмического события, а также удаление нового события от ранее зарегистрированных в ЭПВИ событий. Поэтому эти характеристики очень важны для оценки сейсмической опасности.

Представленные ниже положения могут быть применимы и к другим характеристикам сейсмичности.

Идея самоподобия процесса разрушения в горной породе/земной коре позволяет сформулировать основное предположение о самоподобии сейсмичности следующим образом: сейсмичность в одном ЭПВИ - масштабная версия сейсмичности в другом ЭПВИ. Это подобие не абсолютное, но статистическое, т.е. функция распределения характеристики, рассматриваемой в фиксированном ЭПВИ, является масштабной (сжатой или растянутой по оси ординат) версией базовой функции распределения (Рис. 3) [1-3, 10]. 


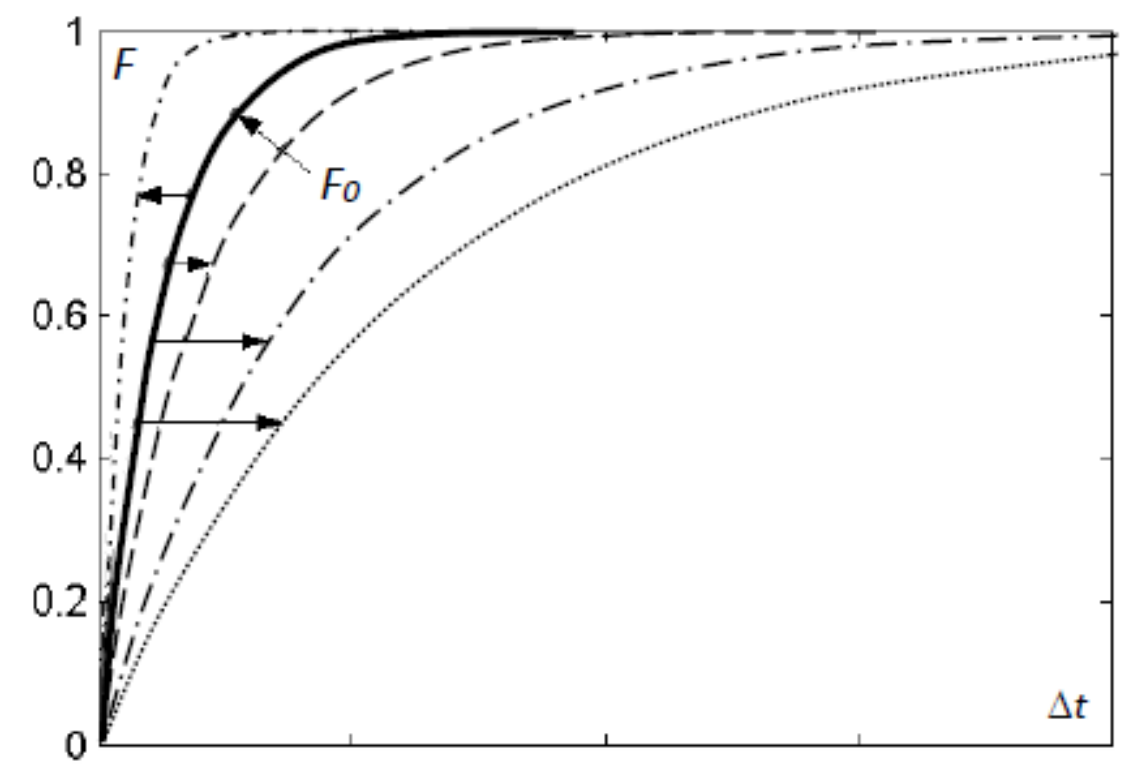

Рис. 3. Масштабирование базовой функции распределения временных интервалов $F_{0}$

Самоподобие структуры сейсмичности означает, что функции распределения какой- либо характеристики сейсмичности для различных ЭПВИ принадлежат к одному и тому же семейству, отличаясь только масштабным коэффициентом, который учитывает параметры ЭПВИ. В частности, он отражает тот факт, что слабые сейсмические события наблюдаются чаще сильных, a также то, что на более протяженной области и за более продолжительный срок регистрируется большее число сейсмических событий. При этом структура сейсмичности, т. е. соотношение числа относительно малых значений рассматриваемой характеристики к относительно большим, должна оставаться постоянной.

Обозначим случайную величину, соответствующую исследуемой характеристике, через V, а соответствующую ей переменную, являющуюся 
аргументом функции распределения и функции плотности вероятности, - через V.

При введенных обозначениях основное утверждение самоподобия сейсмичности может быть сформулировано для функции распределения характеристики:

$$
\left.F(v \mid I)=F(v)=F_{0}(v /<v\rangle^{\prime}\right),
$$

или для соответствующей функции плотности вероятности:

$$
f(v \mid I)=f(v)=\frac{d F(v)}{d v}=\frac{f_{0}\left(v /\langle v\rangle^{\prime}\right)}{\langle v\rangle^{\prime}},
$$

где $\mathrm{F}_{0}$ и $\mathrm{f}_{0}$ - постоянные функции, не зависящие от ЭПВИ; - коэффициент масштабирования для характеристики v, зависящий от параметров интервала I.

Согласно уравнению (1) функция распределения $\mathrm{F}$ является масштабной версией функции F0 (см. Рис. 1). Таким образом, выражение (1) может быть переписано в следующем виде:

$$
V=<v>^{\prime} V_{0},
$$

где - базовая случайная величина, имеющая распределение F0, или случайная величина для ЭПВИ, которому соответствует коэффициент масштабирования . Это означает, что случайная величина $\mathrm{V} /=\mathrm{V} 0$ имеет постоянную функцию распределения $\mathrm{F}_{0}$, не зависящую от масштабного уровня.

Выражения (1) и (2) означают, что распределение F принадлежит к семейству масштабируемых распределений [6]. Однако заметим, что при этом не налагается никаких ограничений на форму базовой функции распределения $\mathrm{F}_{0}$. 
Наиболее часто используемые семейства распределений, являющиеся масштабируемыми, - это экспоненциальное, гамма-распределение, Вейбулла и степенное (без ограничения) [6]. Они нашли достаточно широкое распространение при описании времен ожидания между хронологически последовательными событиями в ЭПВИ. Однако в ряде случаев для этих целей применяют логарифмически-нормальное распределение, но оно не является масштабируемым семейством распределений, что не позволяет описывать с его помощью временную структуру сейсмичности ожидания для различных ЭПВИ.

\section{Общее выражение для коэффициента масштабирования функций}

\section{распределения характеристик сейсмичности}

Вычисление математического ожидания от обеих частей уравнения (3) показывает, что математическое ожидание случайной величины $\mathrm{V}-<\mathrm{V}>$ для некоторого ЭПВИ пропорционально коэффициенту масштабирования : v

$$
\langle V\rangle=\langle v\rangle^{\prime}\left\langle V_{0}\right\rangle=\text { const }\langle v\rangle^{\prime} \text {. }
$$

Следовательно, для получения функции распределения характеристики $\mathrm{F}$ для некоторого ЭПВИ из базовой функции распределения F0 можно использовать коэффициент масштабирования $=$ const $<\mathrm{V}>$.

Согласно уравнению наравне со случайной величиной $\mathrm{V} /=\mathrm{V} 0$ безразмерная случайная величина также будет иметь постоянную функцию распределения, не зависящую от параметров ЭПВИ. v V V

Однако на практике значение математического ожидания неизвестно, хотя оно может быть оценено с помощью выборочного среднего характеристики V для выбранного ЭПВИ, которое является его состоятельной, несмещенной и асимптотически эффективной оценкой. V

Пример построения масштабированных функций распределения времени ожидания $\square \mathrm{T}$ и минимальных расстояний $\square$ Dmin для сейсмичности 
Токтогульского района (на Рис. 4) показывает, что вид функции распределения слабо зависит от выбранных для их построения ЭПВИ. Анализ распределений Т и Dmin других регионов приведен в $[1-3,9,10]$.

Коэффициенты масштабирования могут также определяться через другие соотношения, основанные на модели ускоренных испытаний или закона Гутенберга-Рихтера $[1-4,7-10]$.

Применение теории подобия пространственной и временной структуры сейсмичности открывает ряд новых возможностей. В частности, отклонения от подобия функций распределения $\square \mathrm{T}$ и $\square$ Dmin могут использоваться для выделения сейсмических событий, регистрация которых не представительна [5]. Вариации формы распределения могут также говорить о различных тектонических режимах территорий [4]. Возможны и другие применения разработанной теории.

\section{Заключение}

В статье рассмотрена теория масштабирования функций распределения временных и пространственных характеристик в сейсмологии, соответствующая предположению о подобии структуры сейсмичности в разных энергетическипространственно-временных интервалах.

Проверка разработанной теории и полученных соотношений для различных сейсмоактивных регионов велась для двух характеристик: времени ожидания между землетрясениями $\square \mathrm{T}$ и нового параметра $\square$ Dmin, который является минимальным расстоянием от текущего сейсмического события до ближайшего (в пространстве) события в некотором энергетическопространственно-временном интервале. Распределение $\square \mathrm{T}$ и $\square$ Dmin позволяет оценить временной интервал до следующего землетрясения и удаленность очередного землетрясения от произошедших ранее.

Таким образом, эти характеристики очень важны для оценки сейсмической опасности. 


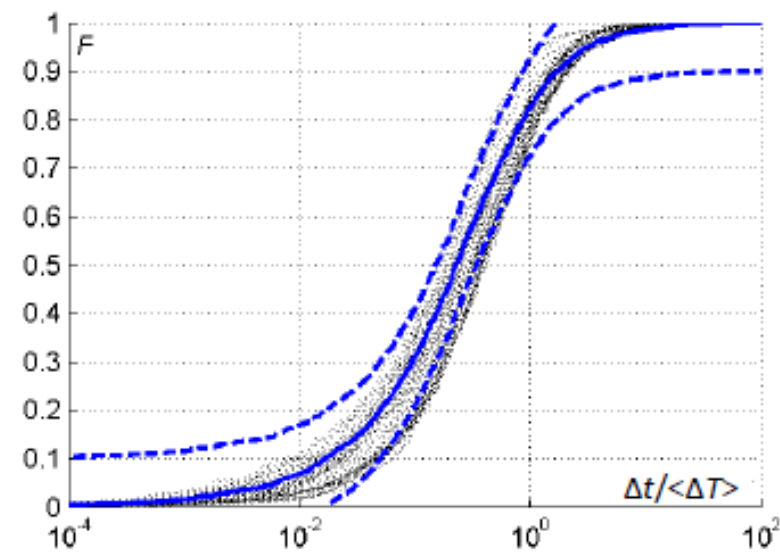

a
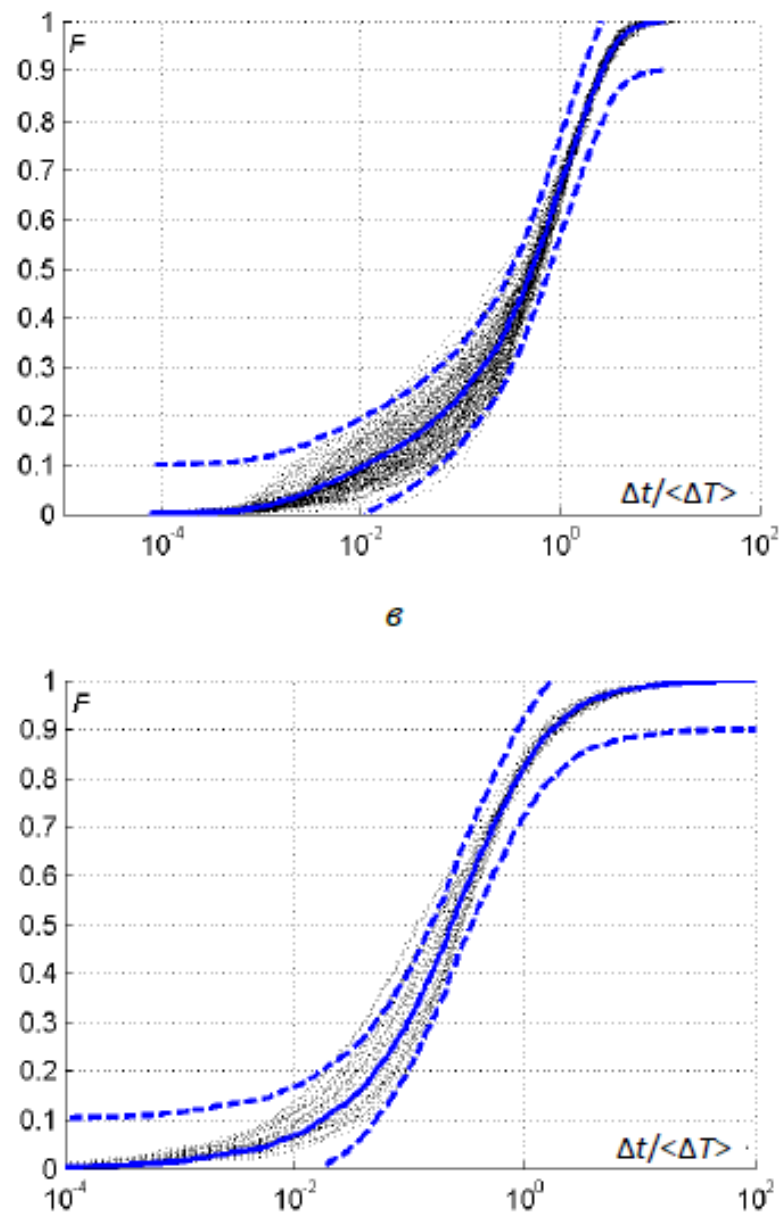

$\partial$

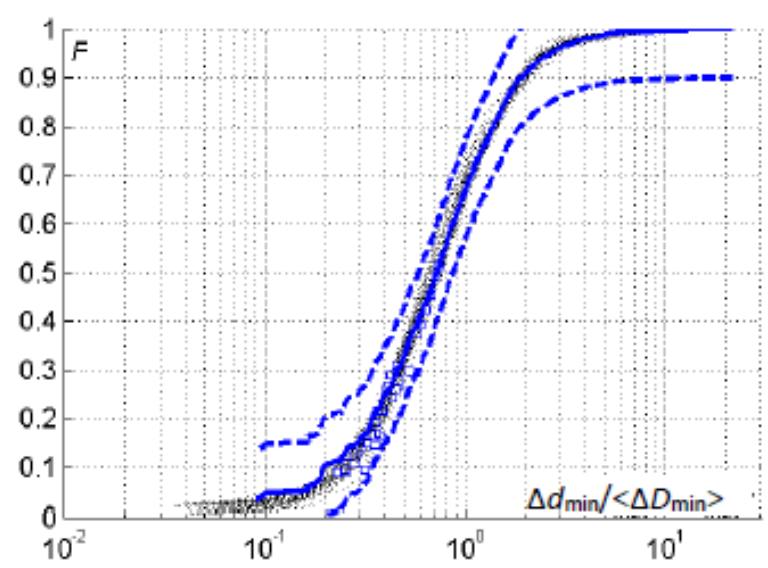

6

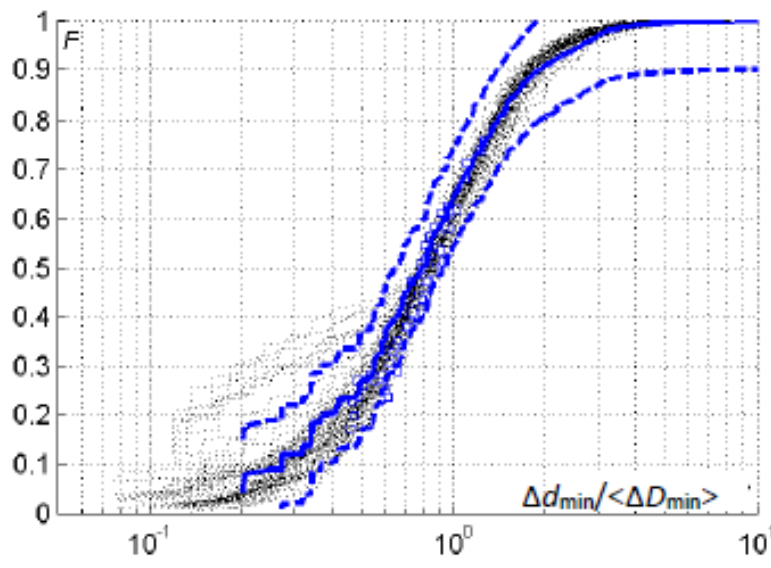

2

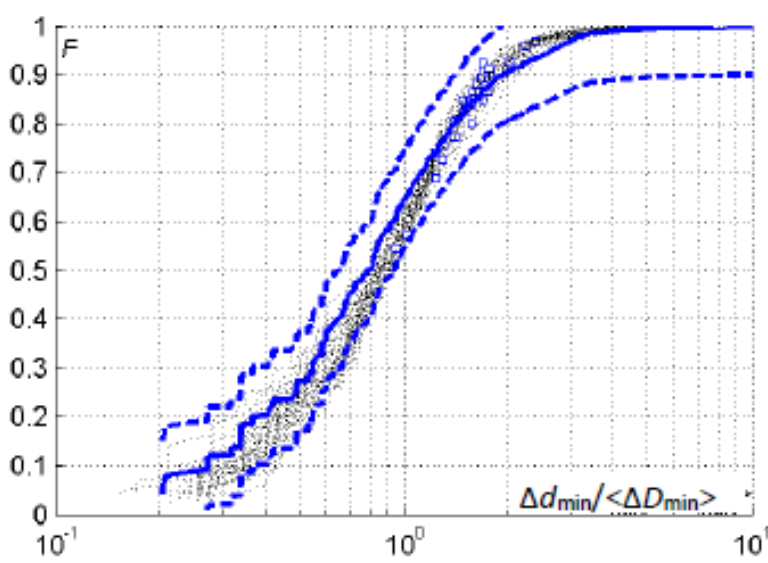

e

Рис. 4. Функции распределения времен ожидания $\Delta T(a, 6, \partial)$ и расстояний $\Delta D_{\min }(6,2, e)$, масштабированные коэффициентами $<\Delta T>_{\text {и }}<\Delta D_{\min }>$ соответственно, для Токтогульского района с изменением параметров ЭПВИ $a, 6-E_{I}$ и $\Delta E_{I} ; \varepsilon, 2-T_{I}$ и $\Delta T_{I} ; \partial, e-X_{I}, Y_{I}$ и $\Delta X_{I}=\Delta Y_{I}=\Delta L_{\downarrow}$ 
Масштабирование распределений с применением основного выражения для коэффициента масштабирования было успешным как для всех энергетическо-пространственно-временных интервалов в случае характеристики $\Delta \mathrm{Dmin}$, так и для различных энергетических диапазонов (масштабных уровней) в случае $\Delta \mathrm{T}$. На примере сейсмичности Токтогульского района показана работоспособность теории, заключающаяся в стабильности масштабированных функций распределения $\Delta \mathrm{T}$ и $\Delta \mathrm{Dmin}$.

Работа выполнена при частичной финансовой поддержке РФФИ (проект № 13-05-00158 А и 14-45-04157 р_сибирь_а).

\section{Список литературы}

1. Герман В.И. Самоподобие временных интервалов между актами разрушения в горных породах на различных масштабных уровнях. // Физические основы прогнозирования разрушения горных пород: Материалы 1-й Международной школы-семинара. (9-15 сент. 2001 г. Красноярск). Красноярск: СибГАУ, 2002. С. 66-72.

2. Герман В.И. Самоподобие временной структуры сейсмических событий на разных масштабных уровнях. // Вулканология и сейсмология. - 2005. - № 3. С. 66-74.

3. Герман В.И. Единая теория подобия структуры сейсмичности: статистический подход. - Красноярск: Сиб. гос. аэрокосмич. ун-т, 2010. - 80 с.

4. Герман В.И. Выделение аномалий структуры сейсмичности и их связь с тектоническим строением территории. // Современная тектонофизика. Методы и результаты. Материалы Второй молодежной школы-семинара. - М.: ИФ3, 2011. Т. 1.

5. Герман В.И. Новый подход к выделению представительных данных в каталогах сейсмических событий. // Современные методы обработки и интерпретации сейсмологических данных. Материалы Шестой Международной сейсмологической школы. - Обнинск: ГС РАН, 2011. С. 69-78.

6. Кокс Д.Р., Оукс Д. Анализ данных типа времени жизни: пер. с англ. - М.: Финансы и статистика, 1988. - $191 \mathrm{c.}$

7. Bak P., Christensen K., Danon L. and Scanlon T. Unified Scaling Law for Earthquakes. // Phys. Rev. Lett. 88, 178501, 2002.

8. Corral A. Long-term clustering, scaling, and universality in the temporal occurrence of earthquakes. // Phys. Rev. Lett. 92, 108501, 2004.

9. German V. Analysis of temporal structures of seismic events on different scale levels // Pure and Applied Geophysics. - 2006. - Vol. 163, № 10. - Pp. 2243-2258.

10. German V.I. Unified scaling theory for distributions of temporal and spatial characteristics in seismology. // Tectonophysics, Vol. 424, № 3-4, 2006. Pp. 167-175. 